\title{
From Bench to Clinic: Accessing Promising Investigational Medications for Patients with HIV Infection in an Urban Family Health Center
}

\author{
John Weiser, MD, MPH, Alice Welch, MPH, RPh, and Neil Calman, MD
}

Background: Patients who experience failure of treatment for human immunodeficiency virus and who have no other treatment options can sometimes obtain promising investigational medications through expanded access programs. However, psychosocial and socioeconomic factors, common among underserved populations, create barriers to enrollment at referral centers. To increase access for unstable patients, an urban family health center developed the capacity to provide investigational medications directly to patients. This article describes that process and the treatment outcomes.

Methods: The necessary steps to implement the program were outlined and accomplished. Patient outcomes were analyzed and the project's value to the organization and its clients was evaluated.

Results: Twenty-five patients received one or more investigational antiretroviral medications. Within 24 weeks of enrollment, the majority of patients achieved undetectable viral levels $(80 \%$ achieved $<400$ copies $/ \mathrm{mL}$ and $64 \%$ achieved $<50$ copies $/ \mathrm{mL})$, with a median decrease of $49,173 \mathrm{copies} / \mathrm{mL}(P=.0002)$ and a median increase in CD4 lymphocyte count of $95 \mathrm{cells} / \mathrm{mm}^{3}(P<.0001)$.

Conclusion: Community health centers can mitigate barriers to access and meet the needs of unstable patients who have no therapeutic options by incorporating access to promising investigational treatments into primary care. The use of centralized institutional review boards can increase efficiency of the review process, but provisions to address local cultural and social issues should be included. ( $\mathrm{J}$ Am Board Fam Med 2010;23:566-570.)

Keywords: HIV, AIDS, Expanded Access, Investigational Drugs, Family Health Center

During the 2 decades after the approval of the first antiretroviral drug, zidovudine, in 1987, 22 medications in 4 classes were licensed for the treatment of HIV. Mortality dropped dramatically and time to clinical progression or death increased among patients who received highly active antiretroviral therapy (HAART). Clinical trials demonstrated sustained viral suppression to $<50$ copies $/ \mathrm{mL}$ in $64 \%$ of pa-

This article was externally peer reviewed.

Submitted 14 November 2009; revised 18 April 2010; accepted 11 May 2010.

From the Institute for Family Health, New York, NY (JW, AW, NC); the Harvard School of Public Health, Boston, MA (JW); and The Graduate Center, City University of New York, New York (AW).

Funding: Grants totaling less than \$2000 for clerical expenses were received from Pfizer and Tibotec.

Conflict of interest: none declared.

Corresponding author: John Weiser, MD, MPH, 21 East 22nd Street, \#8H, New York, NY 10010 (E-mail: jkweiser@gmail.com). tients and to levels of $<400$ copies $/ \mathrm{mL}$ in $71 \%$ of patients after 144 weeks of treatment. ${ }^{1}$

However, despite widespread availability of HAART in the United States and improved effectiveness and tolerability of newer treatment regimens, multidrug resistance leading to treatment failure continued to be a significant problem for patients with long-term HIV infection. ${ }^{2}$ To avoid major AIDS complications, newer treatment options were needed, and in 20063 promising antiretroviral drugs completed phase 3 clinical trials and entered the final Food and Drug Administration (FDA) review process. The new medications could be used in combination and, if licensed, presented a potential opportunity for patients experiencing treatment failure to achieve virologic control and avoid the complications of severe immunodeficiency.

For nearly all such patients, the goal of treatment would shift from maintaining partial viral suppression and slowing the rate of clinical deteri- 
oration, to long-term, full viral suppression with preservation and reconstitution of immune function. This prospect represented the first substantial revision of the paradigm for HIV treatment since the mid-1990s, when the HAART era was heralded by the development of protease inhibitors. Although FDA approval was expected, timing was uncertain and patients who were experiencing treatment failure and who had no other options needed prompt access to the new investigational medication.

After a 1991 report by the Institute of Medicine on expanding access to investigational therapies for HIV infection, ${ }^{3}$ the US Public Health Service established a "parallel track" initiative to provide promising investigational medications to HIV patients who had no therapeutic alternatives while clinical trials to test their effectiveness and safety were conducted. ${ }^{4,5}$ In 2007, timely access to the investigational antiretroviral medications required enrollment in one or more expanded access programs (EAPs), also known as expanded access studies, which were conducted under investigational new drug protocols with requirements for reporting of safety data.

EAPs are typically based at academic research institutions and, for many HIV patients, socioeconomic and psychosocial factors create barriers to enrollment at these facilities. In response, a New York-based community health organization that was providing treatment for a panel of $500 \mathrm{HIV}$ patients made a decision to join the 3 EAPs to provide direct access to the investigational medications for all qualified patients. The experience of community health centers with EAPs has not previously been described, and there are no data comparing outcomes at academic medical centers and community health centers. ${ }^{6}$

The 3 investigational medications were (1) etravirine, $^{7}$ a non-nucleoside reverse transcriptase inhibitor with activity against viruses resistant to other non-nucleoside reverse transcriptase inhibitors; (2) raltegravir, ${ }^{8}$ the first in a new class of integrase inhibitors; and (3) maraviroc, ${ }^{9}$ the first CCR5 coreceptor blocker.

\section{Methods}

\section{operational Issues}

Initial applications were submitted to the pharmaceutical companies that produced each of the 3 investi- gational medications, followed by full applications to the centralized institutional review boards (IRBs) associated with each of the 3 programs. A family physician who fulfilled the New York State Department of Health criteria for designation as an HIV specialist $^{10}$ was assigned to be principal investigator of the programs, and a master's-level health educator who was also trained as a pharmacist was identified to be the program coordinator and assume day-to-day responsibility for all operational aspects. Each centralized IRB required staff training regarding patient recruitment, informed consent, secure record keeping, specimen handling and shipping, and electronic data reporting. Site visits were conducted by the clinical research organizations sponsoring the programs before initiation and periodically for the duration of the program. The organization's malpractice carrier was consulted and it was determined that any untoward outcomes associated with the investigational treatments would be covered under the existing policy.

\section{Patients}

Enrollment was open to any qualifying patient at the center and referrals from outside practices were accepted. Inclusion criteria specified that an effective treatment regimen could not be constructed from among all approved antiretroviral medications because of documented viral resistance or intolerance. Patients were excluded if they had significant hepatic or renal disease or were clinically unstable (as defined by each protocol). Enrollment was permitted in one or more programs, depending on the number of new drugs that would be required to construct a fully suppressive regimen. The intake process included written informed consent obtained by the program coordinator (AW), a history and physical examination performed by the principal investigator (JW), outside record retrieval (for external referrals), and baseline laboratory testing.

Study visits were documented at the initiation of treatment and then at weeks 4, 12, 24, and 36. Receipt and distribution of all medications and the return of any unused drugs were documented in medication logs. Laboratory and other clinical data were entered on secure web sites during each study visit. Any serious adverse events were to be reported electronically and by telephone to the manufacturer and the centralized IRBs. Medical records were clearly labeled to indicate that the patient was receiving investigational medication and what steps to take in the case of possible adverse events. Peri- 
odic progress reports were submitted to the clinical research organizations and centralized IRB for each study.

\section{Statistical Analysis}

Changes in CD4 count and viral load levels while on treatment were tested in an intent-to-treat analysis using the Wilcoxon signed rank test. The association of viral load and CD4 outcomes with sex, race/ethnicity, category of insurance, and participants' referral sources were assessed with multivariate logistic regression. Analyses were performed with Stata software, version 11 (StataCorp, LP, College Station, TX).

\section{Results}

Twenty-five patients were eligible for inclusion and were enrolled in one or more EAPs (Table 1). One patient dropped out before starting treatment but was included in the intent-to-treat analysis. Eleven patients were recruited internally and 14 were referred from outside sources (6 from private practices and 8 from major academic institutions). Twenty-three were eligible for participation because of treatment failure secondary to multidrug resistance and 2 were eligible because of intolerance of available treatment options. Nine patients were non-Hispanic black (36\%), 10 were non-Hispanic white (40\%), and 6 were Hispanic (24\%). The percentage of non-Hispanic white patients was higher among referrals from private practices $(100 \%)$ than from academic centers $(38 \%)$ or internal patients (18\%). Two of the 25 participants were women (one internal and one from an academic center). Median baseline CD4 count was 114 cells $/ \mathrm{mm}^{3}$ (range, 6-524). Median baseline viral load was 43,177 copies/mL (range, $<50-670,000$ ).

Treatment regimens included raltegravir $(\mathrm{n}=$ 4); etravirine $(\mathrm{n}=1)$; raltegravir and etravirine $(\mathrm{n}=18)$; and raltegravir, etravirine, and maraviroc $(n=1)$. If more than one investigational medication was included in the regimen, initiation of treatment was deferred until all medications were available and the full regimen could be started concurrently.

Twenty-four patients completed between 4 and 24 weeks of treatment. Eighty percent (95\% CI, 59-93) reached nadir HIV RNA levels of $<400$ copies $/ \mathrm{mL}$, and $64 \%$ (95\% CI, 43-82) reached a level of $<50$ copies $/ \mathrm{mL}$. Two outlying values of
Table 1. Baseline Characteristics of Participants

\begin{tabular}{|c|c|}
\hline Baseline Variable & n (\%) \\
\hline \multicolumn{2}{|l|}{ Sex } \\
\hline Women & $23(92)$ \\
\hline Men & $2(8)$ \\
\hline \multicolumn{2}{|l|}{ Race/ethnicity } \\
\hline Non-Hispanic black & $9(36)$ \\
\hline Non-Hispanic white & $10(40)$ \\
\hline Hispanic & $6(24)$ \\
\hline \multicolumn{2}{|l|}{ Insurance type } \\
\hline Medicaid & $8(32)$ \\
\hline Medicare & $3(12)$ \\
\hline Commercial & $13(52)$ \\
\hline ADAP & $1(4)$ \\
\hline \multicolumn{2}{|l|}{ Referral source } \\
\hline Internal & $11(44)$ \\
\hline Academic center & $8(32)$ \\
\hline Private practice & $6(24)$ \\
\hline \multicolumn{2}{|c|}{ Baseline labs (median [range]) } \\
\hline CD4 count (cells/mm²) & $114(6-524)$ \\
\hline Viral load (copies/mL) & $54,581(<50-670,000)$ \\
\hline \multicolumn{2}{|l|}{ Referral source } \\
\hline Internal & $11(44)$ \\
\hline Academic center & $8(32)$ \\
\hline Private practice & $6(24)$ \\
\hline \multicolumn{2}{|l|}{ Eligibility criteria } \\
\hline Resistance & $24(96)$ \\
\hline Intolerance & $1(4)$ \\
\hline \multicolumn{2}{|l|}{ Study medications } \\
\hline ETV & $1(4)$ \\
\hline RAL & $4(16)$ \\
\hline ETV/RAL & $19(76)$ \\
\hline ETV/RAL/MVC & $1(4)$ \\
\hline
\end{tabular}

ADAP, New York State AIDS Drug Assistance Program; ETV, etravirine; RAL, raltegravir; MVC, maraviroc.

$>100,000$ copies $/ \mathrm{mL}$ represented a patient who dropped out before starting treatment and another who later reported never having started treatment. Median viral load decrease was 49,173 copies/mL (range, 59,291-669,951; $P<.0001$ ) (Table 2). Median CD4 count among patients being treated was 229 cells $/ \mathrm{mm}^{3}$ (range, $29-515$ cells $/ \mathrm{mm}^{3}$ ). Median CD4 count change was an increase of 95 cells $/ \mathrm{mm}^{3}$ (range, -57 to $333 ; P=.0002$ ). There were no serious adverse events in any of the treatment groups.

Between August 2007 and January 2008, each of the 3 medications received FDA approval, at which time patients were withdrawn from the EAPs and began receiving medications by prescription. Those who were referred from outside facilities received 
Table 2. Median CD4 Lymphocyte Count and Viral Load Levels: Baseline, On Treatment, and Median Change

\begin{tabular}{|c|c|c|c|c|}
\hline & Baseline & On Treatment & Median Change & $P^{*}$ \\
\hline CD4 lymphocyte count (cells $/ \mathrm{mm}^{3}$ ) & $114(6$ to 524$)$ & $229(29$ to 515$)$ & $95(-57$ to 333$)$ & $<.0001$ \\
\hline HIV-1 RNA (copies/mL) & $43,177(<50$ to 670,000$)$ & $<50(<50$ to $>100,000)$ & $-49,173(59,291$ to $-669,951)$ & .0002 \\
\hline
\end{tabular}

*Wilcoxon signed rank test.

their initial prescriptions from the principal investigator (JW), then continued ongoing care from their primary HIV providers.

\section{Discussion \\ Oversight and Monitoring}

Enrollment in the EAPs represented the first experience of our organization and its IRB with complex pharmaceutical protocols. A decision was made by the IRB to issue a waiver permitting use of the centralized IRBs associated with each of the clinical research organizations that were sponsoring the 3 EAPs. The centralized IRBs assumed oversight of human subject protection, including the informed consent process, privacy of information, data collection and reporting, medication storage, and adverse event monitoring; the local IRB maintained oversight of issues specific to incorporating program patients into the workflow of an established practice.

Centralized IRBs conduct reviews of study protocols (in whole or in part) on behalf of all study sites of a multicenter trial that agreed to participate in the centralized process. Examples include the National Cancer Institute centralized IRB, as well as independent review boards that are audited by the FDA, such as the New England IRB. The National Institute of Health supports the use of centralized IRBs for multicenter studies to reduce duplication of effort, delays, and expenses: "The Agency hopes that sponsors, institutions, [IRBs], and clinical investigators involved in multicenter clinical research will consider the use of a single central IRB (centralized IRB review process), especially if using centralized review could improve the efficiency of IRB review." 11 A potential limitation of centralized IRBs is that they may be less attuned to local and cultural issues than are local IRBs. The National Institute of Health guidance statement about the use of centralized IRBs suggests mechanisms by which participating institutions can ensure meaningful consideration of local factors.
Given the resources and time that would have been required for the organization's local IRB to acquire the capacity to oversee 3 complex EAP treatment protocols, use of the centralized IRBs was the only option that would have permitted the undertaking of this initiative. Several institutions in New York City that applied to participate in the EAPs did not use the centralized IRBs and experienced delays of $\geq 2$ months for local IRB processing. Specialists from several academic centers, including an AIDS Clinical Trials Unit, asked to refer patients for earlier enrollment and the requests were accepted. It is important to note that organizations may have specific policies in place regarding the use of centralized IRBs.

\section{Health Equity}

In New York City, 79\% of people living with HIV/ AIDS are not white and $30 \%$ are women, ${ }^{12}$ which is a close approximation of the demographics of the overall HIV population at our center. Nonwhite patients were under-represented in the EAP overall but not among patients who were recruited internally ( $60 \%$ vs $82 \%)$. Women were under-represented by all measures, and the inequality is not likely to be accounted for by a lesser need for new treatment options among women. Further exploration of this occurrence would be appropriate, as would a comparison of the sex and racial/ethnic distribution of the patients enrolled in our program with that of similar programs at academic referral centers.

Quantification of socioeconomic and psychosocial characteristics associated with barriers to access among the participants would provide insight about the value of locating these programs where patients receive primary care. Half of the patients recruited internally were enrolled in Medicaid and more than 4 in 5 were nonwhite; both of these factors are associated with measures of low health status. ${ }^{13,14}$ Nearly two thirds of HIV patients receive treatment for mental illness or substance abuse,${ }^{15}$ which 
can complicate successful treatment of medical illnesses through several pathways.

To justify the investment of resources necessary to carry out a program of this sort, evidence of superior outcomes in a local program relative to the alternative options would be valuable. Studies making that comparison have not been performed, but clearly these patients were able to start treatment earlier than if they had to enroll in programs elsewhere. In addition to the logistic advantages, the use of centralized IRBs expedited the initiation of treatment at our center.

\section{Conclusion}

As new treatments for serious and potentially lifethreatening diseases progress through clinical trials and enter the FDA approval process, there will be a need for unstable patients to have early access to them. Limited access to investigational treatments for serious conditions is likely to contribute to racial/ ethnic and sex disparities in health outcomes. Our experience demonstrates that, despite certain administrative complexities and personnel requirements, community health organizations can successfully incorporate access to investigational treatments into the delivery of primary care and achieve significant clinical benefits for patients with serious illnesses.

The authors would like to acknowledge Diane Hauser, MPA, and Edward Fried, MA, for contributing their organizational expertise.

\section{References}

1. Arribas JR, Pozniak AL, Gallant JE, et al. Tenofovir disoproxil fumarate, emtricitabine, and efavirenz compared with zidovudine/lamivudine and efavirenz in treatment-naive patients: 144-week analysis. J Acquir Immune Defic Syndr 2008;47:74-8.

2. Bhaskaran K, Hamouda O, Sannes M, et al. Changes in the risk of death after HIV seroconversion compared with mortality in the general population. JAMA 2008;300:51-9.

3. Roundtable for the Development of Drugs and Vaccines Against AIDS, Institute of Medicine. Expanding access to investigational therapies for HIV infec- tion and AIDS. Washington, D.C.: The National Academies Press; 1991.

4. Expanded availability of investigational new drugs through parallel track mechanism for people with AIDS and other HIV-related disease-PHS. Notice final policy statement. Fed Regist 1992;57:13250-9.

5. U.S. Department of Health and Human Services, Food and Drug Administration. Access to investigational drugs. Available at http://www.fda.gov/ForConsumers/ ByAudience/ForPatientAdvocates/AccesstoInvestigationalDrugs/ucm176098.htm. Accessed 17 April 2010.

6. Food and Drug Administration, Health and Human Services. Expanded access to investigational drugs for treatment use. Final Rule. Fed Regist 2009;47: 40900-45.

7. Lazzarin A, Campbell T, Clotet B, et al. Efficacy and safety of TMC125 (etravirine) in treatment-experienced HIV-1-infected patients in DUET-2: 24week results from a randomised, double-blind, placebo-controlled trial. Lancet 2007;370:39-48.

8. Steigbigel RT, Cooper DA, Kumar PN, et al. Raltegravir with optimized background therapy for resistant HIV-1 infection. N Engl J Med 2008;359:339-54.

9. Gulick RM, Lalezari J, Goodrich J, et al. Maraviroc for previously treated patients with R5 HIV-1 infection. N Engl J Med 2008;359:1429-41.

10. New York State Department of Health AIDS Institute. HIV specialist policy. Available at http://www. hivguidelines.org/policy/hiv-specialist-policy/. Accessed 17 April 2010.

11. U.S. Department of Health and Human Services, Food and Drug Administration. Guidance for industry. Using a centralized IRB review process in multicenter clinical trials. Available at http://www.fda.gov/RegulatoryInformation/Guidances/ucm127004.htm. Accessed 13 July 2010.

12. New York City Department of Health and Mental Hygiene. New York City HIV/AIDS annual surveillance statistics. Available at http://www.nyc.gov/ $\mathrm{html} / \mathrm{doh} / \mathrm{html} / \mathrm{ah} / \mathrm{hivtables.shtml}$ tables. Accessed 13 July 2010.

13. Berkman LF, Kawachi I. Socioecomic position. In: Social Epidemiology. 1st ed. New York, NY: Oxford University Press; 2000:13-35.

14. Berkman LF, Kawachi I. Discrimination and health. In: Social Epidemiology. 1st ed. New York, NY: Oxford University Press; 2000:36-75.

15. Burnam MA, Bing EG, Morton SC, et al. Use of mental health and substance abuse treatment services among adults with HIV in the united states. Arch Gen Psychiatry 2001;58:729-36. 